Acta Crystallographica Section D

\section{Biological Crystallography}

ISSN 0907-4449

\title{
Structural characterization of a human Fc fragment engineered for lack of effector functions
}

The first three-dimensional structure of a human Fc fragment genetically engineered for the elimination of its ability to mediate antibody-dependent cellmediated cytotoxicity and complement-dependent cytotoxicity is reported. When introduced into the lower hinge and $\mathrm{C}_{\mathrm{H}} 2$ domain of human IgG1 molecules, the triple mutation L234F/L235E/P331S ('TM') causes a profound decrease in their binding to human CD64, CD32A, CD16 and C1q. Enzymatically produced $\mathrm{Fc} / \mathrm{TM}$ fragment was crystallized and its structure was solved at a resolution of $2.3 \AA$ using molecular replacement. This study revealed that the three-dimensional structure of $\mathrm{Fc} / \mathrm{TM}$ is very similar to those of other human $\mathrm{Fc}$ fragments in the experimentally visible region spanning residues 236-445. Thus, the dramatic broad-ranging effects of $\mathrm{TM}$ on $\operatorname{IgG}$ binding to several effector molecules cannot be explained in terms of major structural rearrangements in this portion of the Fc.

\section{Introduction}

The relationship between the biological activity of human immunoglobulin $\mathrm{G}(\mathrm{IgG})$ molecules and their effector functions has been well established. Indeed, efficient antibody-dependent cell-mediated cytotoxicity (ADCC) and complement-dependent cytotoxicity (CDC) have been invoked as crucial determinants in the efficacy of various therapeutic antibodies (Anderson et al., 1997; Green et al., 2000; Cartron et al., 2002; Weng \& Levy, 2003). Conversely, the elimination of antibody effector functions could play a role in decreasing IgG-mediated toxicity events, as shown for the CDC activity of the anti-CD3 monoclonal antibody OKT3 (Xu et al., 2000).

ADCC and CDC are triggered by the binding of various effector molecules to the Fc portion of IgG molecules. Mutational studies have identified several 'hotspots' for Fc binding to the first component of complement activation $\mathrm{C} 1 \mathrm{q}$ and to various $\mathrm{ADCC}$-promoting $\mathrm{Fc} \gamma$ receptors (CD64/32A/16). In particular, lower hinge positions 234 and 235 were shown to have a strong modulating effect on human Fc binding to human CD64 (Canfield \& Morrison, 1991; Chappel et al., 1991; Hezareh et al., 2001), CD32A (Hezareh et al., 2001; Armour et al., 2003), CD16 (Hezareh et al., 2001) and C1q (Xu et al., 2000; Hezareh et al., 2001). Likewise, $\mathrm{C}_{\mathrm{H}} 2$ position 331 was shown to be a major determinant for human IgG binding to human CD64 (Canfield \& Morrison, 1991) and C1q (Tao et al., 1993; Idusogie et al., 2000).

One particular set of substitutions, L234F/L235E/P331S (referred to hereafter as ' $\mathrm{TM}$ '), results in a profound decrease in the binding activity of human IgG1 molecules to human C1q, CD64, CD32A and CD16 (this study). In an effort to shed light on the mechanisms by which TM negatively affects these interactions, we solved the X-ray crystal structure of a human IgG1 Fc fragment containing the TM set of substitutions. This constitutes the first three-dimensional structure of a human Fc fragment specifically engineered for lack of effector functions.

\section{Materials and methods}

\subsection{Reagents, conventions and illustrations}

All chemicals were of analytical grade. Recombinant FLAGtagged human CD32A and CD64 were kindly provided by Linda Xu 
(MedImmune Inc.). Recombinant FLAG-tagged human CD16 (V158 allotype) was generated as previously described (Oganesyan et al., 2008). All antibody amino-acid positions mentioned in the text are identified according to the EU numbering convention described in Kabat et al. (1991). Illustrations were prepared using PyMOL (DeLano Scientific, Palo Alto, California, USA).

\subsection{Generation, expression and purification of $m A b 3649$ and $3649 / \mathrm{TM}$}

A humanized monoclonal antibody directed against human CD19 (referred to hereafter as mAb 3649) was generated at MedImmune Inc. and used as a test molecule for studying the impact of TM on human IgG binding to human effector molecules. The heavy and light chains of mAb $3649(\operatorname{IgG} 1, \kappa)$ were cloned into a previously described mammalian expression vector (Oganesyan et al., 2008). The TM combination of mutations was introduced into the heavy chain of mAb 3649 by site-directed mutagenesis using a QuikChange XL Mutagenesis Kit according to the manufacturer's instructions (Stratagene, La Jolla, California, USA). This generated mAb 3649/TM. Human embryonic kidney (HEK) 293 cells were then transiently transfected with the corresponding antibody constructs and the secreted immunoglobulins were purified using HiTrap protein A columns according to the manufacturer's instructions (GE Healthcare, Piscataway, New Jersey, USA).

\subsection{Analysis of human C1q binding to mAb 3649 and 3649/TM}

In order to characterize the binding of mAb 3649 and 3649/TM to human C1q, the following ELISA was carried out. Individual wells of a 96-well Maxisorp Immunoplate (Nunc, Rochester, New York,
USA) were coated for at least $2 \mathrm{~h}$ at $277 \mathrm{~K}$ with twofold serially diluted samples (mAb 3649 or 3649/TM) at concentrations typically ranging from 100 to $0.05 \mu \mathrm{g} \mathrm{ml}^{-1}$ and then blocked with $4 \%$ milk for at least $2 \mathrm{~h}$ at $310 \mathrm{~K}$. Plates were then successively incubated with $2 \mu \mathrm{g} \mathrm{ml}^{-1}$ human C1q (Quidel, San Diego, California, USA) for $1 \mathrm{~h}$ at $310 \mathrm{~K}$ and sheep anti-human C1q (BioDesign, Saco, Maine, USA; $1 / 1000$ dilution) for $1 \mathrm{~h}$ at $310 \mathrm{~K}$. Incubation with a donkey anti-sheep IgG horseradish peroxidase conjugate (Serotec, Raleigh, North Carolina, USA; 1/10,000 dilution) for $1 \mathrm{~h}$ at room temperature then followed. Horseradish peroxidase activity was detected with TMB substrate (KPL, Gaithersburg, Maryland, USA) and the reaction was quenched with $1 \% \mathrm{H}_{2} \mathrm{SO}_{4}$. Plates were read at $450 \mathrm{~nm}$.

\subsection{Analysis of human Fc $\gamma$ receptors binding to $\mathrm{mAb} 3649$ and 3649/TM}

In order to characterize the binding of mAb 3649 and 3649/TM to human CD64 and CD16/V158, the following ELISA was carried out. Individual wells of a 96-well Maxisorp Immunoplate (Nunc) were coated overnight at $277 \mathrm{~K}$ with a $10 \mu \mathrm{g} \mathrm{ml}^{-1}$ solution of mAb 3649 or $3649 / \mathrm{TM}$ and blocked with $4 \%$ milk for at least $2 \mathrm{~h}$ at $310 \mathrm{~K}$. Incubation with twofold serially diluted samples ( $\mathrm{Fc} \gamma$ receptors) at concentrations typically ranging from 50 to $0.02 \mu \mathrm{g} \mathrm{ml}^{-1}$ for $1 \mathrm{~h}$ at $310 \mathrm{~K}$ then followed. Plates were successively incubated with biotinylated anti-FLAG IgG (Sigma, St Louis, Missouri, USA; 1:100 dilution) and then avidin horseradish peroxidase conjugate (Pierce, Rockford, Illinois, USA; 1:250 dilution) each for $1 \mathrm{~h}$ at $310 \mathrm{~K}$. Plates were developed and read as described above.

Binding of mAb 3649 and 3649/TM to human CD32A was assessed essentially as described above with the following modification: in

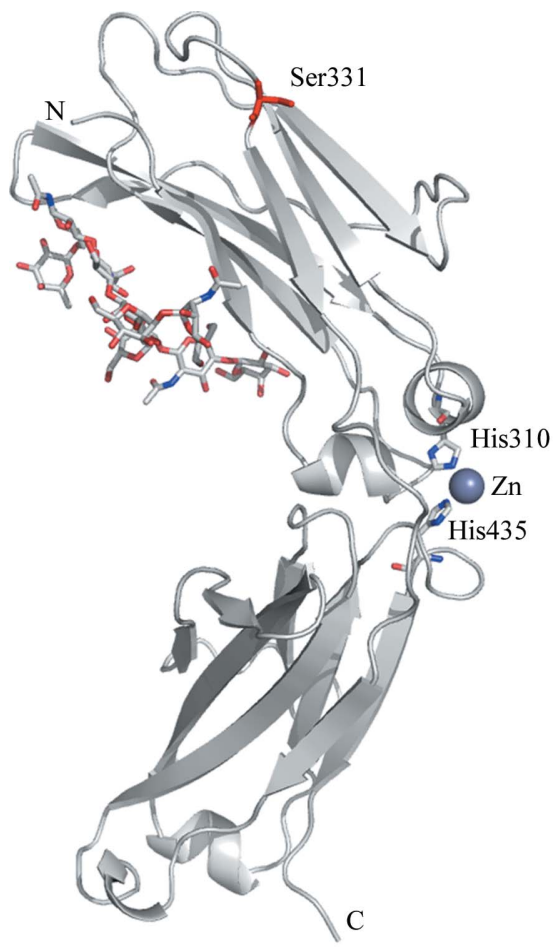

(a)

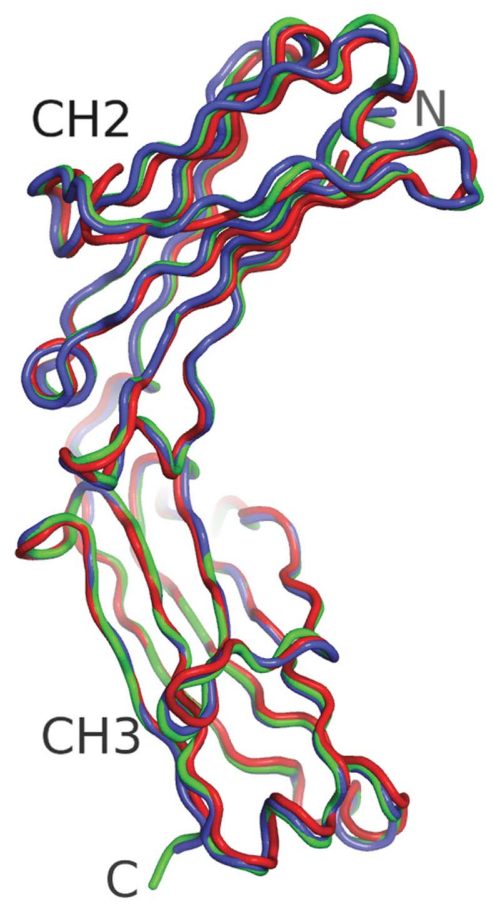

(b)

Figure 1

(a) Representation of the contents of the asymmetric unit of the Fc/TM crystal. The only visible mutation comprising 'TM', P331S, is indicated in red. One zinc ion is shown chelated by two spatially close histidine residues. The conventional 'horseshoe'-shaped homodimeric Fc fragment would be achieved by invoking a crystallographic twofold symmetry operator. The carbohydrate residues attached to Asn 297 were modeled according to their electron density. (b) Stereographic representation of three unliganded human $\mathrm{Fc}$ regions separately superimposed through their respective $\mathrm{C}_{\mathrm{H}} 2$ and $\mathrm{C}_{\mathrm{H}} 3$ domains. Polypeptides are color-coded as follows: blue, 2ql1 (Oganesyan et al., 2008); red, 1h3w (Krapp et al., 2003); green, Fc/TM. 
order to increase the low ELISA signal arising from the poor affinity of the human CD32A-IgG interaction, dilutions of human CD32A were first dimerized by pre-incubation with a biotinylated anti-FLAG IgG (Sigma; 1:100 dilution) for $1 \mathrm{~h}$ at room temperature and then added to the $\mathrm{IgG}$-coated wells.

\subsection{Generation and purification of $\mathrm{Fc} / \mathrm{TM}$}

The human Fc/TM fragment was obtained directly from the enzymatic cleavage of a fully human monoclonal antibody targeting the type I interferon receptor $1(\mathrm{IgG} 1, \kappa)$ and whose Fc contains the $\mathrm{TM}$ set of substitutions. This particular human $\mathrm{IgG}$, which contained an Fc portion identical to that of 3649/TM, was selected for digestion owing to its availability at the time of the experiment. Digestion was carried out using immobilized ficin according to the manufacturer's instructions (Pierce). Purification was first performed on HiTrap protein A columns according to the manufacturer's instructions (GE Healthcare). After dialysis in $50 \mathrm{~m} M$ sodium acetate $\mathrm{pH} 5.2$, the protein solution was applied onto a HiTrap SP HP column (GE Healthcare) and collected in the flowthrough. A final purification step which included loading of this flowthrough onto a HiTrap Q column (GE Healthcare) and elution in an $\mathrm{NaCl}$ gradient yielded a homogenous Fc/TM preparation, as judged by reducing and nonreducing SDS-PAGE. In particular, we note that the Fc/TM SDS-PAGE profile showed the presence of only one band around 25 or $50 \mathrm{kDa}$ under reducing or nonreducing conditions, respectively (data not shown). This observation clearly demonstrated the presence of at least one interchain disulfide bond at positions Cys226 and/or Cys229. Consequently, the mutated 'downstream' residues Phe234 and Glu235 were present in the polypeptide chain comprising the crystal.

\subsection{Crystallization of Fc/TM}

Purified Fc/TM was concentrated to about $5 \mathrm{mg} \mathrm{ml}^{-1}$ using a Centricon concentrator (Millipore, Billerica, Massachusetts, USA; $30 \mathrm{kDa}$ cutoff). Crystallization conditions were identified using the commercial screens from Hampton Research (Hampton Research, Aliso Viejo, California, USA), Emerald BioSystems (Emerald BioSystems Inc., Bainbridge Island, Washington, USA) and Mole-

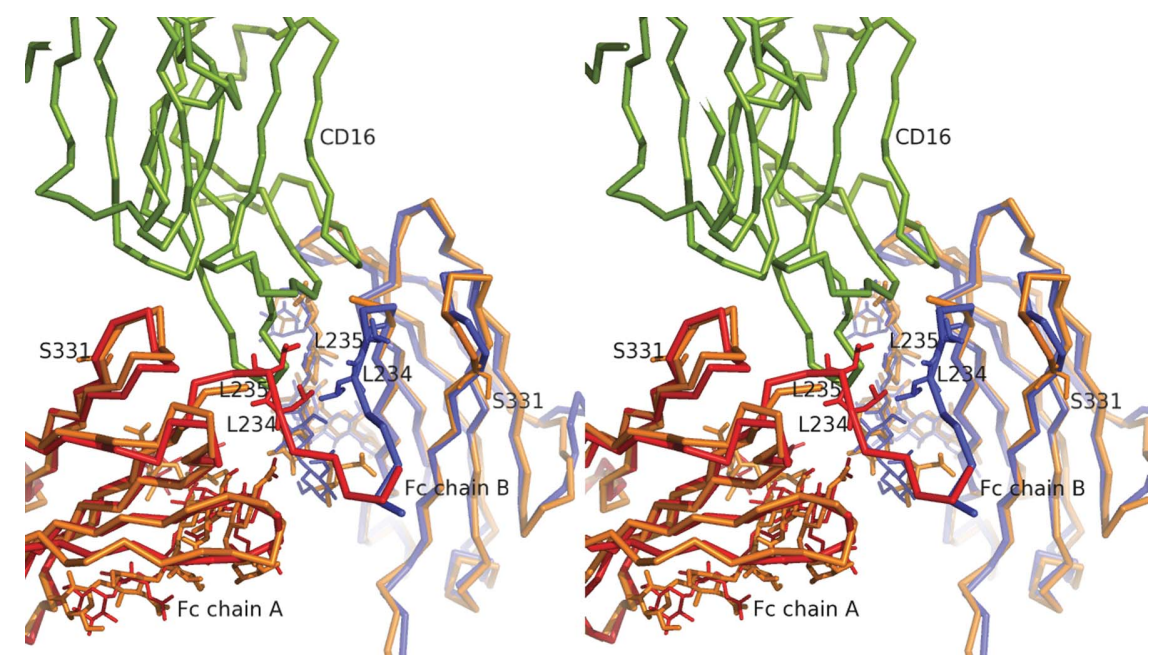

Figure 2

Stereographic superimposition of Fc/TM with the CD16-liganded human $\mathrm{Fc}$ structure corresponding to PDB code 1e4k (Sondermann et al., 2000). Superimposition of $\mathrm{C}^{\alpha}$ atoms of $\mathrm{Fc} / \mathrm{TM}$ and $1 \mathrm{e} 4 \mathrm{k}(\mathrm{CH} 2$ domain residues 236-342) was carried out using LSQKAB (Kabsch, 1976). For both chains of 1e4k, the r.m.s. displacement was estimated at $1.9 \AA$, with a maximum displacement of $6.4 \AA$ for the $\mathrm{C}^{\alpha}$ of residue 286 . Human CD16 and Fc/TM are colored green and orange, respectively. Chains $A$ and $B$ of 1e4k are colored red and blue, respectively. cular Dimensions (Molecular Dimensions Inc., Apopka, Florida, USA). Each screen yielded several potentially usable crystallization conditions. Upon optimization, diffraction-quality crystals were obtained from $0.2 \mathrm{M}$ zinc acetate, $0.1 \mathrm{M}$ imidazole-malate $\mathrm{pH} 8.0,5 \%$ PEG 3350, 5\% glycerol at a protein concentration of $2.0 \mathrm{mg} \mathrm{ml}^{-1}$. Under these conditions, well shaped crystals with three dimensions ranging from 0.1 to $0.2 \mathrm{~mm}$ grew in $2-3 \mathrm{~d}$ (see supplementary Fig. $1^{\mathbf{1}}$ ).

\subsection{Data collection}

Diffraction data were collected from a single crystal at the Center for Advanced Research in Biotechnology (CARB, University of Maryland Biotechnology Institute, Rockville, Maryland, USA) using a Rigaku MicroMax-007 rotating-anode generator with an R-AXIS $\mathrm{IV}^{++}$imaging plate (Rigaku/MSC, The Woodlands, Texas, USA). Prior to cooling, the crystal was kept for a few minutes in its growth solution supplemented with $20 \%$ glycerol. The crystal was then cooled to $105 \mathrm{~K}$ with an X-stream 2000 low-temperature system (Rigaku/MSC). Diffraction to $2.3 \AA$ was achieved after one round of annealing as described in Oganesyan et al. (2008). Diffraction data comprising 234 images were collected using an oscillation range of $0.5^{\circ}$, a crystal-to-detector distance of $200 \mathrm{~mm}$ and an exposure time of $600 \mathrm{~s}$. Data were integrated and scaled using the $H K L-2000$ software (Otwinowski \& Minor, 1997).

\subsection{Structure determination}

Molecular replacement, refinement and electron-density calculations were carried out using the $\mathrm{CCP} 4$ program suite (Collaborative Computational Project, Number 4, 1994). The $C$ face-centered orthorhombic crystal had $58 \%$ solvent content and a $V_{\mathrm{M}}$ of $2.9 \AA^{3} \mathrm{Da}^{-1}$, assuming the presence of one Fc polypeptide in the asymmetric unit of the cell. The crystal structure of Fc/TM was determined by molecular replacement and refined at $2.3 \AA$ resolution. Chain $A$ of the human Fc structure corresponding to PDB entry 2dtq (Matsumiya et al., 2007) was used as the model because of its high resolution and unliganded state. In particular, the $\mathrm{C}_{\mathrm{H}} 2$ and $\mathrm{C}_{\mathrm{H}} 3$ domains were considered separately in order to minimize any bias in terms of the relative conformation of the domains. Data to $3.0 \AA$ resolution were used to solve the molecularreplacement problem using Phaser (McCoy et al., 2005). After refinement of the solutions, the final LL gain and the $Z$ score were 1192 and 31, respectively. Weighted electron density calculated with FWT/PHWT at $3.0 \AA$ showed a good match to the model, with minor differences in some loops of the $\mathrm{C}_{\mathrm{H}} 2$ domain. Strong positive difference electron density calculated with DELFWT/PHDELWT was visible in the expected position of $\mathrm{N}$-linked carbohydrate residues attached to Asn297. There was no density present for any hinge residue preceding that at position 236, a result presumably attributable to the high flexibility of this region. To this effect, we note that only two previously described unliganded human Fc structures revealed positions 234 and 235 (PDB codes 2dtq and 2dts; Matsumiya et al., 2007). Likewise, the

\footnotetext{
${ }^{\mathbf{1}}$ Supplementary material has been deposited in the IUCr electronic archive (Reference: MH5013). Services for accessing this material are described at the back of the journal.
} 
Table 1

$\mathrm{X}$-ray data-collection and model-refinement statistics.

Values in parentheses are for the highest resolution shell.

\begin{tabular}{ll}
\hline Wavelength $(\AA)$ & 1.54 \\
Resolution $(\AA)$ & $36.83-2.30(2.38-2.30)$ \\
Space group & $C 222_{1}$ \\
Unit-cell parameters $(\AA)$ & $a=50.18, b=147.30$, \\
& $c=75.47$ \\
Total reflections & 54409 \\
Unique reflections & 12617 \\
Average redundancy & $4.31(2.72)$ \\
Completeness $(\%)$ & $98.3(90.0)$ \\
$R_{\text {merge }}$ & $0.062(0.300)$ \\
$I / \sigma(I)$ & $13.0(3.3)$ \\
$R$ factor/free $R$ factor & $0.216 / 0.275$ \\
R.m.s.d. bonds $(\AA)$ & 0.012 \\
R.m.s.d. angles $\left({ }^{\circ}\right)$ & 1.48 \\
Residues in most favored region of $(\varphi, \psi)$ space $\dagger(\%)$ & 89.9 \\
Residues in additionally allowed region of $(\varphi, \psi)$ space $(\%)$ & 10.1 \\
No. of protein atoms & 1678 \\
No. of nonprotein atoms & 189 \\
$B$ factor (model/Wilson) $\left(\AA^{2}\right)$ & $43 / 40$ \\
&
\end{tabular}

$\dagger$ The Ramachandran plot was produced using PROCHECK (Laskowski et al., 1993).

residues at positions 446 and 447 could not be visualized. The residue at position 331 was first modeled as an alanine.

Several alternating rounds of refinement with REFMAC5 (Murshudov et al., 1997) and manual building using the $O$ graphics software (Jones et al., 1991) converged with an $R$ factor of 21.6 and a free $R$ factor of 27.5 for data to $2.3 \AA$ resolution. After the first round of refinement, the electron density allowed placement of the carbohydrates as well as substitution by a serine residue at position 331 . In later stages of refinement, the model was analyzed using the $T L S$

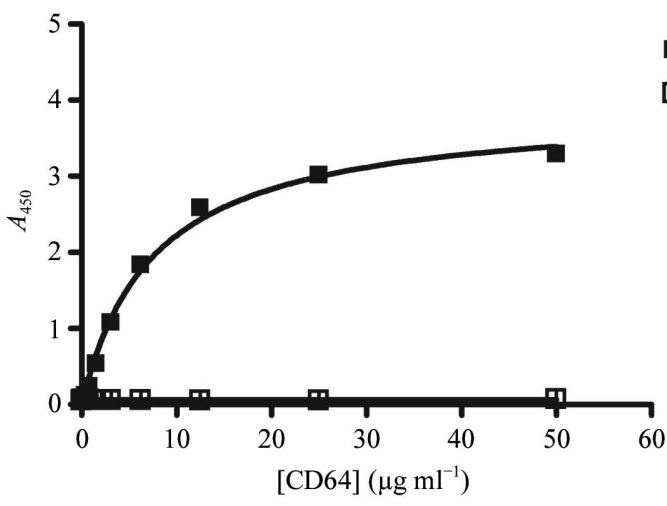

(a)

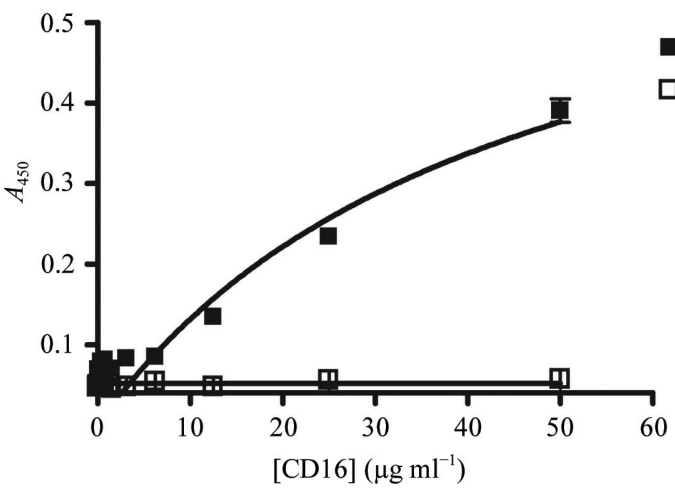

(c)

3649

3649/TM
Motion Determination (TLSMD) program running on its web server (Painter \& Merritt, 2006a,b). Further refinement was then carried out with REFMAC5 in TLS and restrained refinement mode using five distinct groups of residues (236-324, 325-341, 342-358, 359-403 and 404-445). Zinc ions present in the crystallization buffer were detected in the electron density and modeled as such when the coordination sphere and distance permitted. In particular, one zinc ion was found to be coordinated by His 310 and His 435 . Another was coordinated by His 285 and His 268 of the symmetry-related polypeptide. Two others were bound to Glu318 and Glu345. In all cases, water molecules completed the expected tetrahedral coordination sphere of the zinc ions. The carbohydrate moiety was modeled according to its electron density and the final model contained nine sugar residues, essentially as described by us in the context of another human Fc structure (PDB code 2q11; Oganesyan et al., 2008). The final model contained 75 solvent molecules. Crystallographic data and refinement statistics are given in Table 1.

\section{Results and discussion}

$\mathrm{Fc} / \mathrm{TM}$ crystallized in space group $C 222_{1}$, with one polypeptide in the asymmetric unit (Fig. 1a). The crystal diffracted to $2.3 \AA$ resolution and exhibited a relatively high average mosaicity of $1.26^{\circ}$. This high mosaicity appeared to be a property of both cooled and noncooled crystals. All residues at positions $236-445$ could be traced in the electron density. As mentioned above, no electron density was observed for hinge residues prior to position 236. Thus, although present in the crystal (see \$2), both the L234F and L235E mutations were invisible. As expected, the electron density at position 331 corresponded to serine (see supplementary Fig. 2).

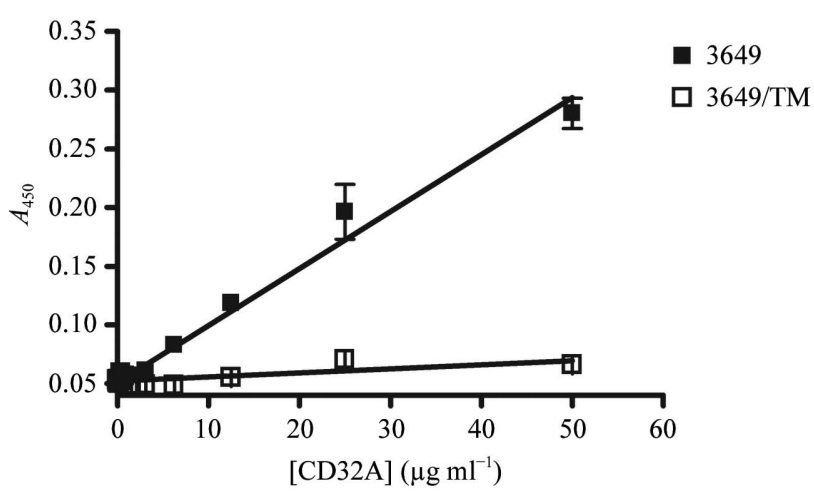

(b)

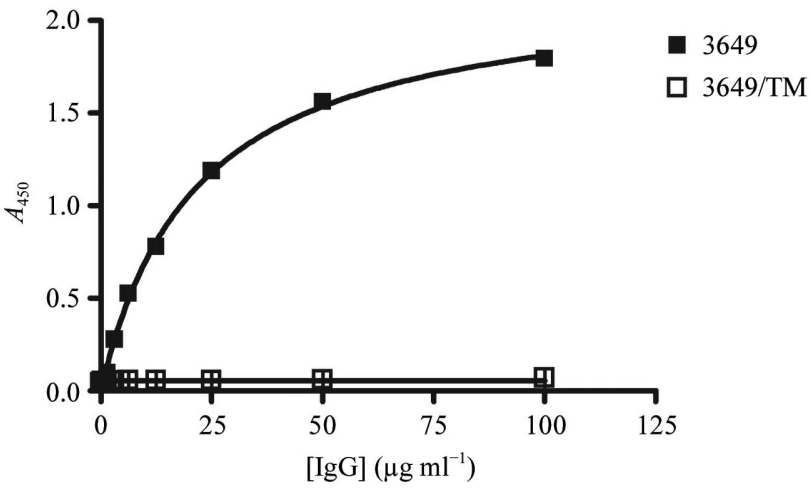

(d)

Figure 3

Analysis of the binding of human (a) CD64, (b) CD32A, (c) CD16/V158 and (d) C1q to mAb 3649 and 3649/TM. Measurements were carried out using an ELISA format as described in $\$ 2$. Errors were estimated as the standard deviations of at least two individual measurements for each interacting pair. The illegibility of the error bars for some data points is the direct result of the corresponding very low standard deviations. Data shown are representative of at least two independent series of experiments. 
Table 2

Structural comparison of three unliganded human Fc structures.

\begin{tabular}{lll}
\hline Molecule pair & Residue span & R.m.s.d. $(\AA)$ \\
\hline Fc/TM $\dagger-2 q 11 \ddagger$ & $236-444\left(\mathrm{C}_{\mathrm{H}} 2 / \mathrm{C}_{\mathrm{H}} 3\right)$ & 0.51 \\
Fc/TM-1h3w $\$$ & $238-444\left(\mathrm{C}_{\mathrm{H}} 2 / \mathrm{C}_{\mathrm{H}} 3\right)$ & 1.27 \\
2ql1-1h3w & $238-444\left(\mathrm{C}_{\mathrm{H}} 2 / \mathrm{C}_{\mathrm{H}} 3\right)$ & 1.29 \\
Fc/TM-2q11 & $236-342\left(\mathrm{C}_{\mathrm{H}} 2\right)$ & 0.61 \\
Fc/TM-2q11 & $342-444\left(\mathrm{C}_{\mathrm{H}} 3\right)$ & 0.36 \\
Fc/TM-1h3w & $238-342\left(\mathrm{C}_{\mathrm{H}} 2\right)$ & 1.58 \\
Fc/TM-1h3w & $342-444\left(\mathrm{C}_{\mathrm{H}} 3\right)$ & 0.43 \\
2ql1-1h3w & $238-342\left(\mathrm{C}_{\mathrm{H}} 2\right)$ & 1.58 \\
2q11-1h3w & $342-444\left(\mathrm{C}_{\mathrm{H}} 3\right)$ & 0.37 \\
\hline
\end{tabular}

$\dagger$ This study (PDB code 3c2s). $\quad$ Oganesyan et al. (2008). § Krapp et al. (2003).

The overall three-dimensional structure of Fc/TM was very similar to the previously reported structures of unliganded human Fc regions (Deisenhofer, 1981; Krapp et al., 2003; Matsumiya et al., 2007; Oganesyan et al., 2008). More precisely, the human Fc structures corresponding to PDB codes $1 \mathrm{~h} 3 \mathrm{w}$ (Krapp et al., 2003) and 2ql1 (Oganesyan et al., 2008) were closest to Fc/TM in terms of unit-cell parameters, asymmetric unit content, space group and packing. The extent of this similarity was evident when the three structures were superimposed (Fig. 1b). Corresponding r.m.s.d. values are shown in Table 2. This overall structural conservation extended to a previously described human Fc in complex with human CD16 (PDB code 1e4k; Sondermann et al., 2000), including the intermolecular contact interface (see Fig. 2).

The introduction of TM into the Fc of human IgG1s resulted in an almost complete loss of their binding to several effector molecules (CD64, CD32A, CD16 and C1q) as shown in Fig. 3. In addition, the three-dimensional structure of $\mathrm{Fc} / \mathrm{TM}$ was found to be very similar to that of other liganded and unliganded human Fc fragments in the region spanning residues 236-445 (see above). Thus, we conclude that the dramatic broad-ranging functional effects of the TM set of substitutions were not caused by major structural rearrangements in this portion of $\mathrm{Fc} / \mathrm{TM}$. Although no definitive statement can be made for the 'unseen' positions 234 and 235, we hypothesize that these effects may be mediated by the localized loss of a few interactions at the mutation sites. However, we cannot rule out that one or more of the mutations described in this work might also trigger major structural changes in other unseen parts of the Fc, such as in the lower or middle hinge regions.

\section{References}

Anderson, D. R., Grillo-Lopez, A., Varns, C., Chambers, K. S. \& Hanna, N. (1997). Biochem. Soc. Trans. 25, 705-708.

Armour, K. L., van de Winkel, J. G., Williamson, L. M. \& Clark, M. R. (2003). Mol. Immunol. 40, 585-593.

Canfield, S. M. \& Morrison, S. L. (1991). J. Exp. Med. 173, 1483-1491.

Cartron, G., Dacheux, L., Salles, G., Solal-Celigny, P., Bardos, P., Colombat, P. \& Watier, H. (2002). Blood, 99, 754-758.

Chappel, M. S., Isenman, D. E., Everett, M., Xu, Y. Y., Dorrington, K. J. \& Klein, M. H. (1991). Proc. Natl Acad. Sci. USA, 88, 9036-9040.

Collaborative Computational Project, Number 4 (1994). Acta Cryst. D50, 760-763.

Deisenhofer, J. (1981). Biochemistry, 20, 2361-2370.

Green, M. C., Murray, J. L. \& Hortobagyi, G. N. (2000). Cancer Treat. Rev. 26, 269-286.

Hezareh, M., Hessell, A. J., Jensen, R. C., van de Winkel, J. G. \& Parren, P. W. (2001). J. Virol. 75, 12161-12168.

Idusogie, E. E., Presta, L. G., Gazzano-Santoro, H., Totpal, K., Wong, P. Y., Ultsch, M., Meng, Y. G. \& Mulkerrin, M. G. (2000). J. Immunol. 164, 41784184.

Jones, T. A., Zou, J.-Y., Cowan, S. W. \& Kjeldgaard, M. (1991). Acta Cryst. A47, 110-119.

Kabat, E. A., Wu, T. T., Perry, H. M., Gottesman, K. S. \& Foeller, C. (1991). Sequences of Proteins of Immunological Interest, 5th ed. Bethesda: NIH Publications.

Kabsch, W. (1976). Acta Cryst. A32, 922-923.

Krapp, S., Mimura, Y., Jefferis, R., Huber, R. \& Sondermann, P. (2003). J. Mol. Biol. 325, 979-989.

Laskowski, R. A., MacArthur, M. W., Moss, D. S. \& Thornton, J. M. (1993). J. Appl. Cryst. 26, 283-291.

McCoy, A. J., Grosse-Kunstleve, R. W., Storoni, L. C. \& Read, R. J. (2005). Acta Cryst. D61, 458-464.

Matsumiya, S., Yamaguchi, Y., Saito, J., Nagano, M., Sasakawa, H., Otaki, S., Satoh, M., Shitara, K. \& Kato, K. (2007). J. Mol. Biol. 368, 767-779.

Murshudov, G. N., Vagin, A. A. \& Dodson, E. J. (1997). Acta Cryst. D53, 240-255.

Oganesyan, V., Damschroder, M. M., Leach, W., Wu, H. \& Dall'Acqua, W. (2008). Mol. Immunol. 45, 1872-1882.

Otwinowski, Z. \& Minor, W. (1997). Methods Enzymol. 276, 307-326.

Painter, J. \& Merritt, E. A. (2006a). Acta Cryst. D62, 439-450.

Painter, J. \& Merritt, E. A. (2006b). J. Appl. Cryst. 39, 109-111.

Sondermann, P., Huber, R., Oosthuizen, V. \& Jacob, U. (2000). Nature (London), 406, 267-273.

Tao, M. H., Smith, R. I. \& Morrison, S. L. (1993). J. Exp. Med. 178, 661667.

Weng, W. K. \& Levy, R. (2003). J. Clin. Oncol. 21, 3940-3947.

Xu, D., Alegre, M. L., Varga, S. S., Rothermel, A. L., Collins, A. M., Pulito, V. L., Hanna, L. S., Dolan, K. P., Parren, P. W., Bluestone, J. A., Jolliffe, L. K. \& Zivin, R. A. (2000). Cell. Immunol. 200, 16-26. 